\title{
Thermal rectification in bulk materials with asymmetric shape
}

\author{
D. Sawaki, ${ }^{1}$ W. Kobayashi, ${ }^{2,3, a)}$ Y. Moritomo, ${ }^{2}$ and I. Terasaki ${ }^{4}$ \\ ${ }^{1}$ Department of Physics, Waseda University, Tokyo 169-8555, Japan \\ ${ }^{2}$ Graduate School of Pure and Applied Sciences, University of Tsukuba, Ibaraki 305-8571, Japan \\ ${ }^{3}$ PRESTO, Japan Science and Technology Agency, Saitama 332-0012, Japan \\ ${ }^{4}$ Department of Physics, Nagoya University, Aichi 464-8602, Japan
}

(Received 30 November 2010; accepted 5 February 2011; published online 25 February 2011)

\begin{abstract}
We investigate thermal rectification in a bulk material with a pyramid shape to elucidate shape dependence of the thermal rectification, and find that rectifying coefficient $R$ is 1.35 for this shape, which is smaller than $R=1.43$ for a rectangular shape. This result is fully duplicated by our numerical calculation based on Fourier's law. We also apply this calculation to a given shape, and show a possible way to increase $R$ depending on the shape. (C) 2011 American Institute of Physics. [doi:10.1063/1.3559615]
\end{abstract}

A thermal rectifier is a device in which heat flows in a forward direction, while it hardly flows in the opposite direction. Owing to controllability of the heat current, the thermal rectifier can be applied to phononic devices as a diode is essential for electronic devices. Since the first report on thermal rectification at an interface between $\mathrm{Cu}$ and $\mathrm{Cu}_{2} \mathrm{O}$, ${ }^{1}$ this research field has been developed by both experimental and theoretical studies. ${ }^{2}$ In particular, several advanced studies concerning a discovery of thermal rectification in nonuniformly mass-loaded carbon nanotube ${ }^{3}$ and a prediction of thermal rectification in anharmonic one-dimensional lattice model/quantum thermal systems ${ }^{4-7}$ have been reported in 2000s, which gives renewal interests to this research field. After these studies, thermal transistor, ${ }^{8}$ thermal memory, ${ }^{9}$ and thermal logic gate ${ }^{10}$ are proposed as possible applications of the thermal rectifier.

Recently, we have demonstrated thermal rectification of an oxide thermal rectifier as a bulk effect ${ }^{11}$ according to a theory based on Fourier's law. ${ }^{12}$ This theory predicts thermal rectification in a sample made of two different materials with different temperature dependences of thermal conductivities. According to Fourier's law,

$$
J=-\kappa[x, T(x)] \frac{d T(x)}{d x},
$$

heat flux $J$ is proportional to thermal conductivity $\kappa$. Since $\kappa$ is a function of position $x$ and absolute temperature $T(x)$, the averaged thermal conductivity $\kappa_{\mathrm{av}}$ of the sample may change depending on a direction of the temperature gradient under finite temperature difference $\Delta T$ (see Fig. 1 of Ref. 11 or Ref. 12). Thus, the heat flux in a forward direction $\left(J_{f}\right)$ can be different from that in the reverse direction $\left(J_{r}\right)$ through Eq. (1) leading to $R>1$, where $R$ represents the thermal rectifying coefficient defined by a ratio of the heat fluxes in the forward and reverse directions $\left(R \equiv\left|J_{f}\right| /\left|J_{r}\right|\right)$. We have found $R=1.43$ at $40 \mathrm{~K}$ with $\Delta T=60 \mathrm{~K}$ in the thermal rectifier made of $\mathrm{LaCoO}_{3}$ and $\mathrm{La}_{0.7} \mathrm{Sr}_{0.3} \mathrm{CoO}_{3}{ }^{11}$

There are several strategies to achieve larger $R$ in bulk materials as follows. (1) To utilize an interface between two materials with different phonon bands. In this case, $R$ is predicted to be up to $2000 .^{13}$ (2) To provide materials which

${ }^{\text {a)} E l e c t r o n i c ~ m a i l: ~ k o b a y a s h i . w a t a r u . g f @ u . t s u k u b a . a c . j p . ~}$ exhibits structural phase transition accompanying a rapid change in $\kappa$ at the transition temperature. (3) To form a thermal rectifier with a proper shape. It is theoretically predicted that carbon nanohorn and carbon nanoribbon exhibit thermal rectification because of larger heat current from a larger section to the smaller section. ${ }^{9-18}$ This kind of the shape effect is also possible in bulk materials according to Eq. (1). However, experimental studies on shape-dependent thermal rectification have not been reported yet. In this letter, we will address the issue (3).

Polycrystalline samples of $\mathrm{LaCoO}_{3}$ (LCO) and $\mathrm{La}_{0.7} \mathrm{Sr}_{0.3} \mathrm{CoO}_{3}$ (LSCO) were prepared by a solid state reaction. Stoichiometric amounts of $\mathrm{La}_{2} \mathrm{O}_{3}, \mathrm{SrCO}_{3}$, and $\mathrm{Co}_{3} \mathrm{O}_{4}$ were thoroughly mixed, and calcined at $1273 \mathrm{~K}$ for $24 \mathrm{~h}$. The products were finely ground, filled up in latex tubes with a small amount of water to increase sample's porosity, and pressed at $40 \mathrm{MPa}$. Then, the pressed sample was calcined at $1273 \mathrm{~K}$ for $24 \mathrm{~h}$. X-ray diffraction pattern was measured using a standard diffractometer with $\mathrm{Fe} K \alpha$ radiation. Any impurity peaks were not detected. A mass density of the sample is evaluated to be $50.7 \%$ of the ideal density for LCO and $54.5 \%$ for LSCO, respectively.

Our measurement system is depicted as a schematic figure in Fig. 1(a). First, LCO and LSCO sample bars were cut to let them have a pyramidlike shape. The sectional area and length of the samples are shown in the right panel of Fig. 1(a). Then, the samples were bonded by silver paste with high thermal conductivity (Kyocera Chemical CT285), and annealed at $423 \mathrm{~K}$ for $0.5 \mathrm{~h}$ and at $493 \mathrm{~K}$ for $1.5 \mathrm{~h}$ to dry the paste. To make heat current $I_{\mathrm{h}}$ into the rectifier, a resistive heater with $r=120 \Omega$ was put on the top of the rectifier together with a copper plate at point (5). Another copper plate was attached on the bottom of the rectifier and the plate was glued to the cold head of closed cycle refrigerator by GE varnish (GE7031). Chromel-constantan thermocouples were attached at points (1)-(4) to monitor temperatures in the presence of the heat current. Detailed description of the measurement system is given in the previous reports. ${ }^{11,19}$ Thermal conductivity was measured by a conventional steady state technique using chromel-constantan differential thermocouple. As shown in Fig. 1(b), $\kappa$ for LCO and LSCO is five times smaller than that of a single crystal, ${ }^{20}$ respectively, which is attributed to the high porosity of the samples. This low thermal conductivity is important to maintain the large 
(a)
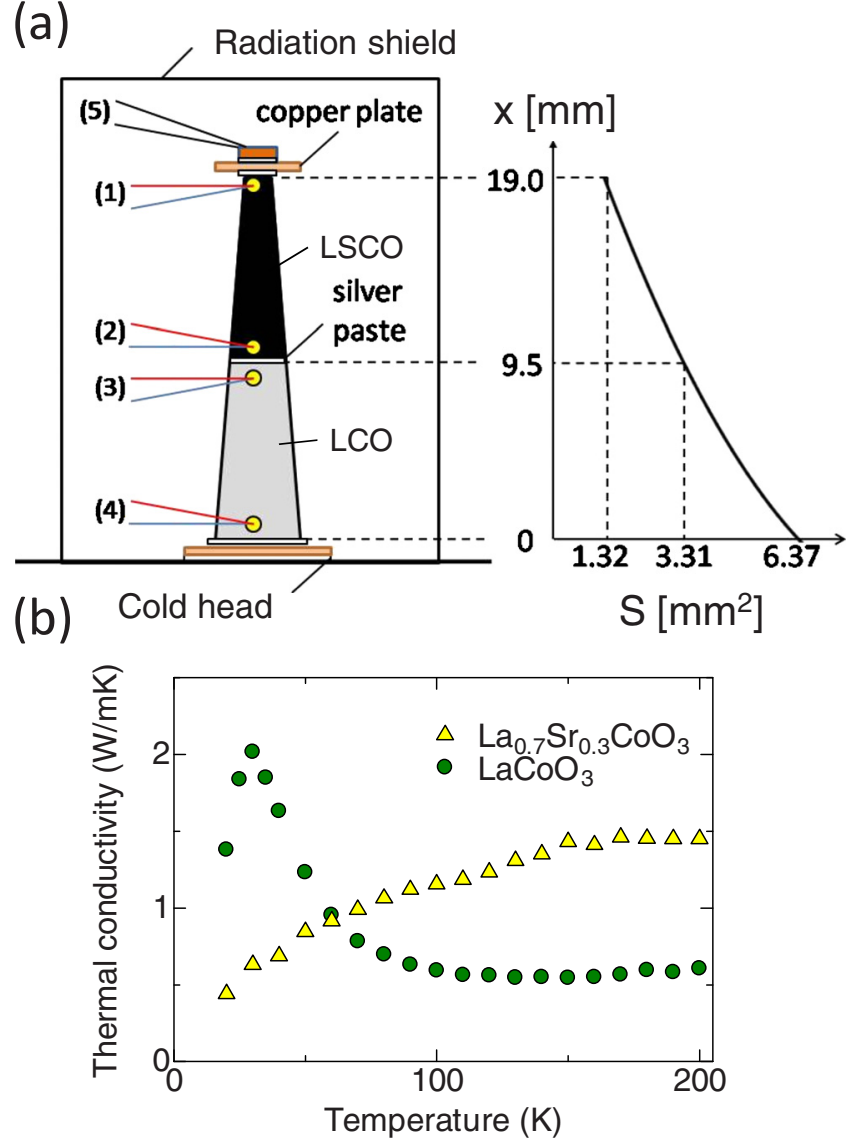

FIG. 1. (Color online) (a) Schematic figure of the thermal rectifier (LSCOtop configuration). (1)-(4) represent chromel-constantan thermocouples and (5) resistive heater. $S(x)$ indicates sectional area of the thermal rectifier. (b) Temperature dependence of the thermal conductivity of $\mathrm{LaCoO}_{3}$ and $\mathrm{La}_{0.7} \mathrm{Sr}_{0.3} \mathrm{CoO}_{3}$ polycrystalline samples.

temperature difference between points (1) and (4).

Figures 2(a) and 2(b) show the monitored temperatures as a function of time at points (1)-(4) in the forward direction (LSCO-top configuration) and the reverse direction (LCO-top configuration), respectively. With increasing electric current $I$, the temperatures systematically increase. The heat current $I_{\mathrm{h}}$ caused by the electric current through the relationship of $I_{\mathrm{h}}=r I^{2}$ is controlled to keep 70, 80,90, and $100 \mathrm{~K}$ at point (1). The most striking feature is that the magnitude of the heat current in the forward direction $\left(I_{\mathrm{hf}}\right)$ is significantly larger than that in the reverse direction $\left(I_{\mathrm{hr}}\right)$ showing the thermal rectification. The temperature difference between points (3) and (4) is completely explained by the thermal conductances of LCO and LSCO, which ensures that contact thermal resistance at the interface between LCO and LSCO is negligible. Note that contact thermal resistance between the rectifier and the cold head is also negligibly small.

We evaluate this experimental results using Fourier's law. From Eq. (1), the temperature distribution of the thermal rectifier is derived as

$$
\begin{aligned}
& T(x)=T(0)-\int_{0}^{x} \frac{I_{\mathrm{h}}(\xi)}{\kappa[\xi, T(\xi)] S(\xi)} \mathrm{d} \xi, \\
& I_{\mathrm{h}}(x)=I_{\mathrm{h}}(0)-\sigma \int_{0}^{x} l(\xi)\left\{T(\xi)^{4}-T_{\mathrm{C}}^{4}\right\} d \xi,
\end{aligned}
$$

where $I_{\mathrm{h}}, S, \sigma, l$, and $T_{C}$ represent heat current, sectional area, Stefan-Boltzmann constant, length of periphery of the (a)

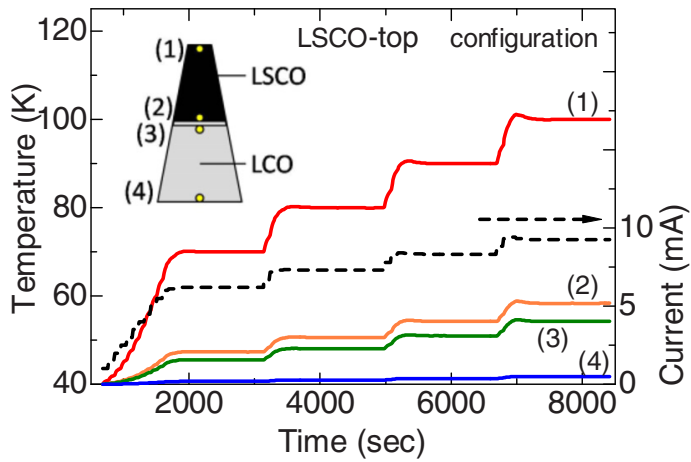

(b)

(c)
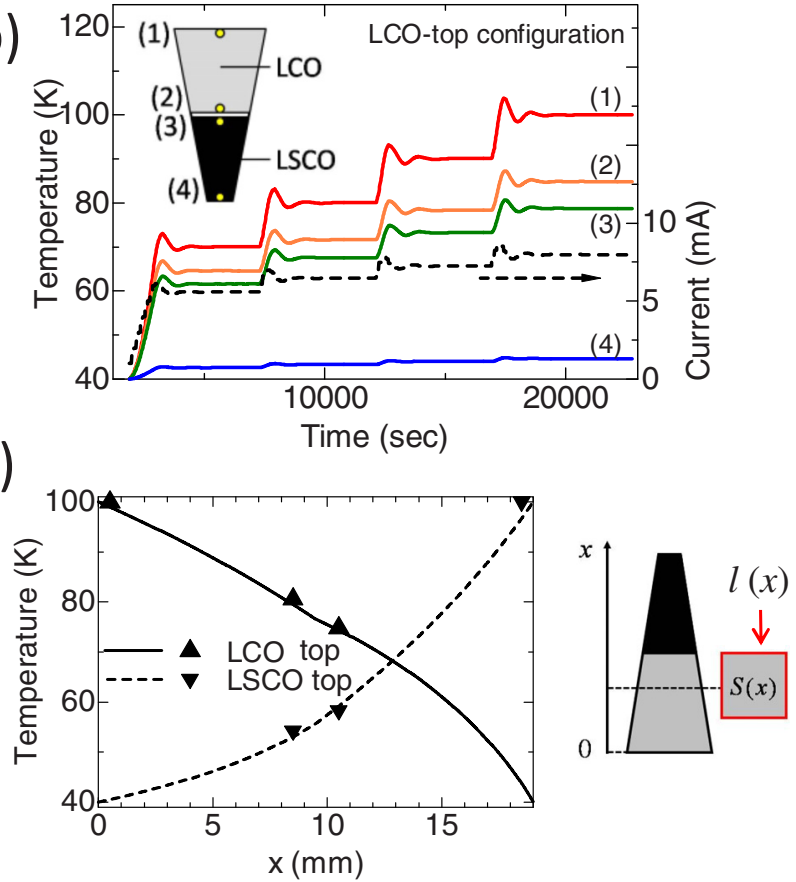

FIG. 2. (Color online) Monitored temperatures as a function of time at points (1)-(4) of (a) LCO-top and (b) LSCO-top configurations, respectively. (c) Temperature distributions in the thermal rectifier with LCO-top and LSCO-top configuration, respectively. Solid and dotted lines represent numerically calculated temperature distribution by Eq. (2). $S(x)$ and $l(x)$ are sectional area and the length of periphery of $S(x)$, respectively.

sectional area $S$, and temperature of the cold head (40 K), respectively. The radiation loss is properly treated in the second line of Eq. (2). It should be emphasized that $I_{\mathrm{h}}(0)$ is only parameter for calculating the temperature distribution under a boundary condition decided by the experiments. Here, as the boundary condition, temperatures at point (1) $(x=0 \mathrm{~mm})$ and point $(4)(x=1.9 \mathrm{~mm})$ were set at $100 \mathrm{~K}$ and $40 \mathrm{~K}$, respectively. As $\kappa[x, T(x)], S(x)$, and $l(x)$, experimental data shown in Fig. 1 were used. As shown in Fig. 2(c), calculated temperature distribution of the rectifier is in good agreement with the experimental data, which shows that the heat current in the pyramid-shaped rectifier is consistently explained by Fourier's law.

Figure 3(a) shows the calculated and experimental heat currents $I_{\mathrm{hf}}(0)$ (LSCO-top configuration) and $I_{\mathrm{hr}}(0)$ (LCO-top configuration) as a function of temperature difference $\Delta T$ $\equiv T(x=0 \mathrm{~mm})-T(x=1.9 \mathrm{~mm})$. With increasing $\Delta T, I_{\mathrm{h}}$ monotonically increases, and experimental data are well reproduced by the calculation. As shown in Fig. 3(b), the rectifying coefficient as a ratio of $I_{\mathrm{hf}}(0)$ and $I_{\mathrm{hf}}(0)$ is 1.35 at $\Delta T=60 \mathrm{~K}$, which is rather small compared with $R=1.43$ of the rectangular one. 


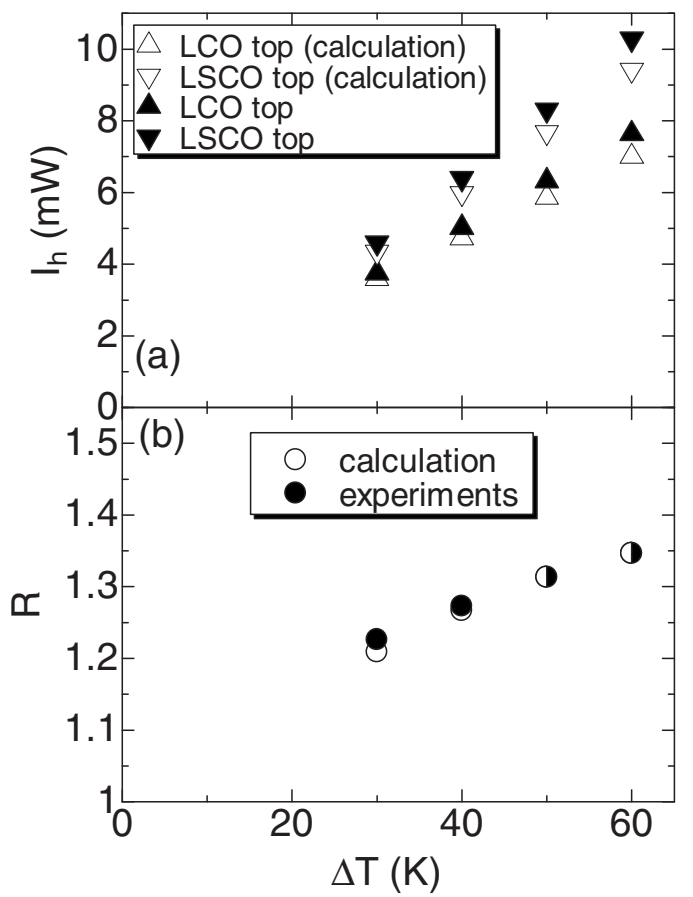

FIG. 3. Calculated and measured (a) heat currents and (b) the rectifying coefficient $R$ of both configurations.

To address how $R$ depends on the thermal rectifier's shape, we perform numerical calculation of $R$ in a sample made of two different homogeneous materials with "a given shape." Figure 4(a) shows two equivalent thermal rectifiers

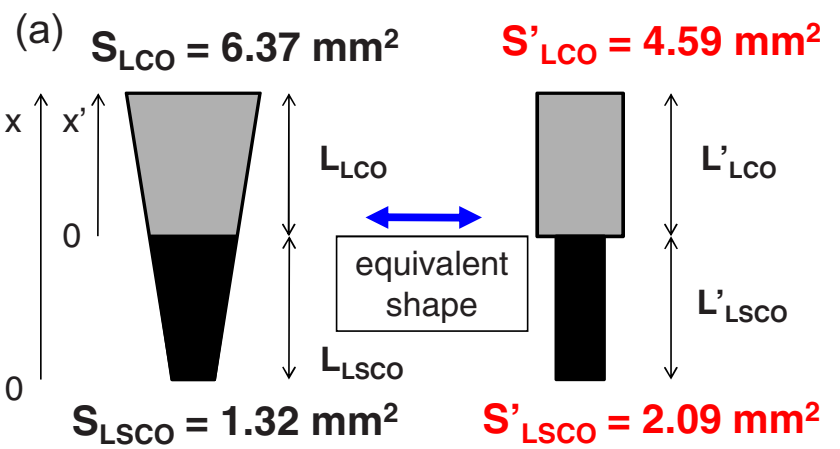

(b)

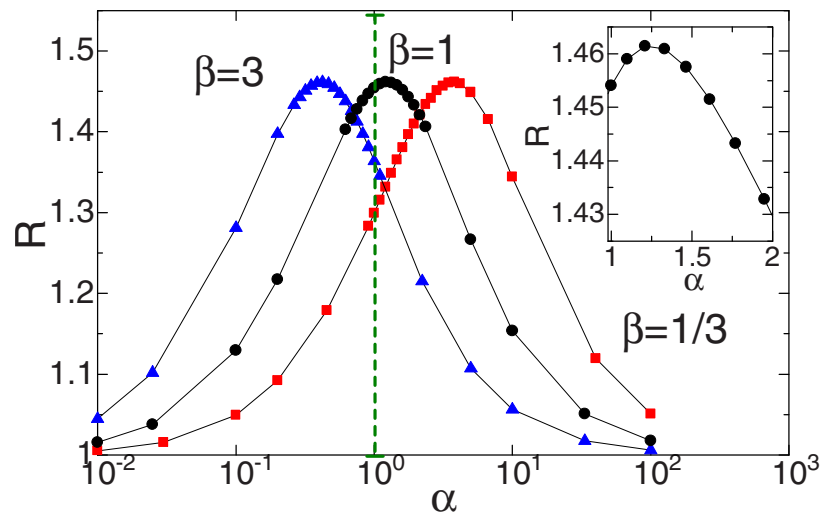

FIG. 4. (Color online) (a) Two equivalent thermal rectifiers which show same calculation result of $I_{\mathrm{hf}}(0), I_{\mathrm{hr}}(0)$, and $R$ under same boundary condition. (b) Calculated $R$ as a function of $x$ and $y$. The inset shows the enlarged calculated result in the range of $1 \leq x \leq 1.5,1.425 \leq R \leq 1.465$. which show same calculation results of $I_{\mathrm{hf}}, I_{\mathrm{hr}}$, interface temperature $\left(T_{\text {int }}\right)$ between LCO and LSCO, and $R$ under same boundary condition. To convert from a pyramid shape (a given shape) to an equivalent rectangular shape, a simple analytical relation is applied,

$$
\frac{L^{\prime}}{S^{\prime}}=\int_{0}^{L} \frac{d x}{S(x)},
$$

where $L\left(L^{\prime}\right)$ and $S\left(S^{\prime}\right)$ represent a length and a sectional area of a given shape (equivalent rectangular shape), respectively.

Figure 4(b) shows the calculated $R$ as a function of $\alpha$ and $\beta$, where $\alpha \equiv S_{\mathrm{LSCO}}^{\prime} / S_{\mathrm{LCO}}^{\prime}\left(S_{\mathrm{LSCO}}^{\prime}, S_{\mathrm{LCO}}^{\prime}\right.$ : sectional areas of LSCO and LCO with the equivalent rectangular shape), and $\beta \equiv L_{\mathrm{LSCO}}^{\prime} / L_{\mathrm{LCO}}^{\prime}\left(L_{\mathrm{LSCO}}^{\prime}, L_{\mathrm{LCO}}^{\prime}\right.$ : lengths of LSCO and LCO with the equivalent rectangular shape). In the case of $\beta=1, R$ takes the maximum of 1.46 at $\alpha=1.2$. Thus, $R$ increases by $0.7 \%$ compared with a rectifier with $\alpha=1$ and $\beta$ $=1$. Depending on $\beta, R$ profile shifts on $x$ axis, which is easily understood by thinking the thermal conductance of the thermal rectifier with rectangular shape. By applying this study to other materials, the thermal rectification may further enhance. We believe that this study will give a possible route to increase rectifying coefficient.

We have investigated shape-dependent thermal rectification from both experimental and theoretical approaches. The cobalt oxide thermal rectifier with pyramidlike shape exhibits the rectifying coefficient of 1.35 , which is rather small compared with that of rectangular-shaped thermal rectifier. The temperature distribution, heat current, and rectifying coefficient are consistently reproduced by Fourier's law. We have also shown that equivalent thermal rectifier with rectangular shape can be derived by a simple analytical relation, and found tiny enhanced rectifying coefficient at $\alpha=1.2$ and $\beta=1$. We believe that this study will give a possible route to increase rectifying coefficient.

This study was partly supported by the Murata Science Foundation.

${ }^{1}$ C. Starr, J. Appl. Phys. 7, 15 (1936).

${ }^{2}$ For example, see the introduction of C. Dames, J. Heat Transfer 131, 061301 (2009).

${ }^{3}$ C. W. Chang, D. Okawa, A. Majumdar, and A. Zettl, Science 314, 1121 (2006).

${ }^{4}$ M. Terraneo, M. Peyrard, and G. Casati, Phys. Rev. Lett. 88, 094302 (2002).

${ }^{5}$ B. Li, L. Wang, and G. Casati, Phys. Rev. Lett. 93, 184301 (2004).

${ }^{6}$ J.-P. Eckmann and C. Mejía-Monasterio, Phys. Rev. Lett. 97, 094301 (2006)

${ }^{7}$ N. Yang, N. Li, L. Wang, and B. Li, Phys. Rev. B 76, 020301(R) (2007).

${ }^{8}$ B. Li, L. Wang, and G. Casati, Appl. Phys. Lett. 88, 143501 (2006).

${ }^{9}$ L. Wang and B. Li, Phys. Rev. Lett. 101, 267203 (2008).

${ }^{10}$ L. Wang and B. Li, Phys. Rev. Lett. 99, 177208 (2007).

${ }^{11}$ W. Kobayashi, Y. Teraoka, and I. Terasaki, Appl. Phys. Lett. 95, 171905 (2009).

${ }^{12}$ M. Peyrard, Europhys. Lett. 76, 49 (2006).

${ }^{13}$ B. Li, J. Lan, and L. Wang, Phys. Rev. Lett. 95, 104302 (2005).

${ }^{14}$ N. Yang, G. Zhang, and B. Li, Appl. Phys. Lett. 93, 243111 (2008).

${ }^{15} \mathrm{G}$. Wu and B. Li, J. Phys.: Condens. Matter 20, 175211 (2008).

${ }^{16}$ N. Yang, G. Zhang, and B. Li, Appl. Phys. Lett. 95, 033107 (2009).

${ }^{17}$ J. Hu, X. Ruan, and Y. P. Chen, Nano Lett. 9, 2730 (2009).

${ }^{18}$ J. W. Jiang, J. S. Wang, and B. Li, Europhys. Lett. 89, 46005 (2010).

${ }^{19}$ W. Kobayashi, Y. Teraoka, and I. Terasaki, J. Electron. Mater. 39, 1488 (2010).

${ }^{20}$ K. Berggold, M. Kriener, C. Zobel, A. Reichl, M. Reuther, R. Muller, A. Freimuth, and T. Lorenz, Phys. Rev. B 72, 155116 (2005). 